\section{\$44. Effects of Non-axisymmetric Magnetic Field on Characteristics of Axisymmetric Cusp DEC}

Tomita, Y.,

Yasaka, Y., Takeno, H. (Kobe Univ.),

Ishikawa, M., Nakashima, Y., Katanuma, I., Cho, T.

(Univ. Tsukuba)

In order to investigate the characteristics of the cusp DEC, a single slant cusp configuration. The oblique uniform magnetic field is applied to the cusp DEC with axisymmetric field as a perturbation,

$$
\vec{B}_{1}=B_{1} \sin \alpha \vec{x}-B_{1} \cos \alpha \vec{z},
$$

where $\alpha$ is the angle of the oblique magnetic field from the axisymmetric axis $z$. This magnetic field leads to the perturbed vector potential,

$$
A_{1 y}=B_{1} \cos \alpha x-B_{1} \sin \alpha z \text {. }
$$

One of the indices to investigate the effect of the perturbed field to the axisymmetric DEC is Störmer potential $V_{s t}$ for $j$-th plasma species,

$$
\begin{aligned}
V_{s t}=\frac{m_{j}}{2} & r^{2} \dot{\theta}^{2} \\
& =\frac{1}{2 m_{j} r^{2}}\left[P_{\theta}-q_{j} \psi-q_{j} \frac{\partial\left(v_{y} A_{1 y}\right)}{\partial \dot{\theta}}\right]^{2} \\
& \simeq V_{s t 0}(r, z)+V_{s t 1}(r, \theta, z) .
\end{aligned}
$$

Here $P_{\theta}$ and $\psi$ are the canonical angular momentum and the flux function, respectively. The unperturbed and perturbed Störmer potentials are expressed as

$$
\begin{aligned}
V_{s t 0}(r, z)= & \frac{1}{2 m_{j} r^{2}}\left(P_{\theta 0}-q_{j} \psi\right)^{2}, \text { and } \\
V_{s t 1}(r, \theta, z)= & -\frac{q_{j} B_{1}\left(P_{\theta 0}-q_{j} \psi\right) r \cos \theta}{m_{j} r^{2}} \\
& \times(r \cos \theta \cos \alpha-z \sin \alpha),
\end{aligned}
$$

respectively. $P_{\theta 0}$ is the unperturbed canonical angular momentum, which is one of the constant of motions. The contour lines of the unperturbed Störmer potentials $V_{s t 0}$ for ions are shown in Fig.1 for the case of the canonical angular momentum $P_{\theta} /\left(e \mu_{0} I_{c, \text { in }} R_{\text {in }} / \pi\right)=0.5$, where $I_{c, i n}$ and $R_{i n}$ are the coil current and radius of the inlet single cusp coil, respectively. In Fig. 1 the radius and current of the outlet coil are double of the inlet one, which makes the slant cusp field. The labels in Fig.1 indicate the values of $V_{s t 0} /\left[\left(e \mu_{0} I_{c, i n} / \pi\right)^{2} / 2 m_{i}\right]$. The stagnation point $\left(\partial V_{s t 0}\left(r, z ; P_{\theta}\right) / \partial r=\partial V_{s t 0}\left(r, z ; P_{\theta}\right) / \partial z=0\right.$ of the Störmer potential in $(r, z)$ space give classification between trapped charged particles in the line cusp region and those which are passed through the single cusp field to the point cusp region. The value of Störmer potential at the stagnation in Fig.1 is 1.55. The perturbation of the non-axisymmetric field changes the distribution of the Störmer potential. The azimuthal dependence of the perturbed Störmer potential is shown in Fig. 2, where the perturbed magnetic field $B_{1} /\left(\mu_{0} I_{c, \text { in }} / \pi R_{\text {in }}\right)=1.0$ and $\alpha=10$ degree. One can see the non-axisymmetric magnetic field deforms the barrier of potential, where the ions with lower energy cannot pass through the enhanced potential to the point cusp. Roughly speaking, one percent perturbation of the magnetic field changes the potential by one percent at the most.

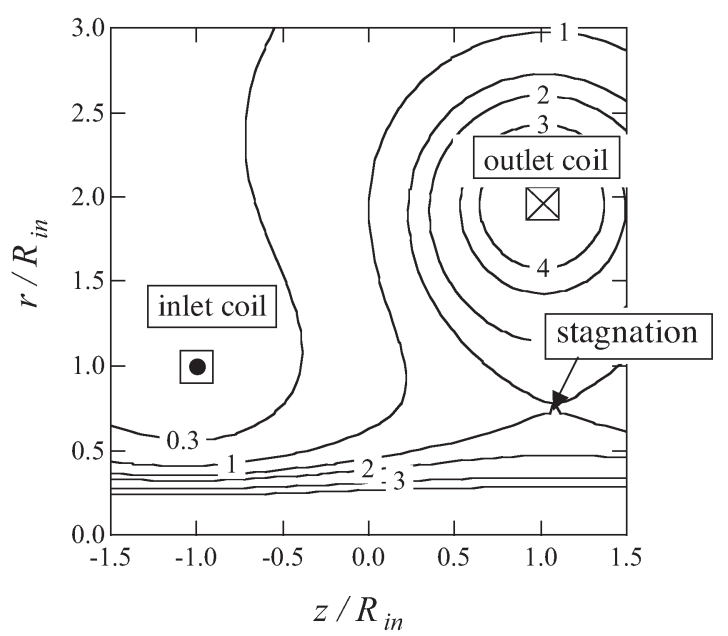

Fig. 1. Contour lines of the unperturbed Störmer potentials for ions. The normalized potential at the stagnation point is 1.55 .

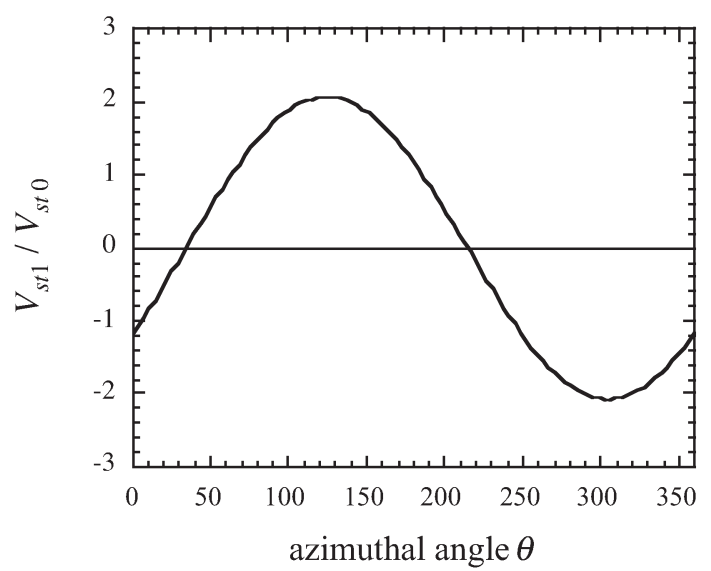

Fig.2. Azimuthal dependence of the perturbed Störmer potential. 